\title{
Background and Technical Studies for GENIUS as a Dark Matter Experiment
}

\author{
L. Baudis, G. Heusser, B. Majorovits, Y. Ramachers, \\ H. Strecker and H. V. Klapdor-Kleingrothaus \\ Max-Planck-Institut für Kernphysik, Heidelberg, Germany
}

\begin{abstract}
The GENIUS project is a proposal for a new dark matter detector, with an increased sensitivity of three orders of magnitude relative to existing direct dark matter detection experiments. We performed a technical study and calculated the main background sources for the relevant energy region in a detailed detector geometry. The achieved overall background level and detector performance confirm the outstanding potential of GENIUS as a powerful tool for the direct search of WIMPs in our Galaxy.
\end{abstract}

\section{Introduction}

GENIUS (GErmanium in liquid NItrogen Underground Setup) is a proposal for operating a large amount of 'naked' Ge detectors in liquid nitrogen for dark matter and alternatively $\beta \beta$-decay researches [1],2,3, 3], with an improved sensitivity of three orders of magnitude relative to present experiments. The idea to operate ionization HPGe detectors directly in liquid nitrogen has been already discussed in [5]. In [6] it has been shown, that the detectors work reliably under such conditions. If the detector performance in liquid nitrogen is unaltered or even better than for usual Ge detectors, the liquid can act both as a cooling medium and as an effective shielding against external radioactivity, since liquid nitrogen can be processed to a very high purity. The proposed scale of the experiment would be a nitrogen tank of about $12 \mathrm{~m}$ diameter and $12 \mathrm{~m}$ height which contains in its dark matter version $100 \mathrm{~kg}$ (40 detectors) of natural germanium suspended in its center. The optimal location would be the Gran Sasso Underground Laboratory.

To cover large parts of the MSSM parameter space, relevant for the detection of neutralinos as the dark matter candidate [7,8], a maximum background level of $10^{-2}$ counts/( $\mathrm{kg}$ y $\mathrm{keV}$ ) in the energy region between $0-100 \mathrm{keV}$ has to be achieved. This means a very large further background reduction in comparison to our recent best result (20.82 counts/(kg y keV)) 9] with an enriched detector of the Heidelberg-Moscow experiment [10] and to all other running dark matter experiments (for a review see [4]). 


\section{Technical study}

To demonstrate the feasibility of operating Ge detectors in liquid nitrogen, instead of in a vacuum-tight cryostat system [11], a first experiment has been successfully performed in the low level laboratory in Heidelberg with one naked p-type Ge crystal immersed in a $50 \mathrm{l}$ dewar [3]. Already in this attempt we could not see any deterioration in the detector performance relative to our conventionally operated detectors.

In a second phase the goal was to look for possible interferences between two or more naked Ge crystals, to test different cable lengths between FETs and crystals and to design and test a preliminary holder system of high molecular polyethylene. We started a technical study operating three germanium detectors on a common plastic holder system inside liquid nitrogen. All crystals were of p-type and weighted about $300 \mathrm{~g}$ each.

A picture of the three-crystal holder-system can be seen in figure 1 . Two thin polyethylene plates $(1 \mathrm{~cm}$ thick $)$ are used to fix the contacts to the crystals. The FETs are placed close to the liquid nitrogen surface but kept inside. Cables having three different lengths $(2,4$ and $6 \mathrm{~m})$ connect the three crystals to their FETs.

The main purpose of the experiment was to test the behaviour of the crystals in the low energy region: energy resolution, energy threshold and possible signs of microphonic events caused by nitrogen boiling. The general performance of the crystals is as stable as already seen with a single detector inside liquid nitrogen. We couldn't observe any cross talk using only p-type detectors (same polarity for the HV-bias), since cross talk signals have the wrong polarity and are filtered by the amplifier.

Figure 2 shows a background spectrum and figure 3 a ${ }^{133} \mathrm{Ba}$ calibration spectrum of one of the naked Ge detectors in liquid nitrogen. The cable length between detector and FET was $6 \mathrm{~m}$ (winded up in loops). We achieved ab energy energy resolution of $1.0 \mathrm{keV}$ at $300 \mathrm{keV}$ and a threshold of $2 \mathrm{keV}$. No microphonic events due to nitrogen boiling beyond $2 \mathrm{keV}$ could be detected. We conclude that the performance of the Ge detectors is as good (or even better) as for conventionally operated crystals, even with $6 \mathrm{~m}$ cable lengths between crystal and FET.

\section{Background considerations}

The aim of the GENIUS experiment is to reach the extremely low background level of 0.01 events/( $\mathrm{kg} \mathrm{y} \mathrm{keV})$ in the energy region below $100 \mathrm{keV}$. To show that this is indeed achievable, we performed detailed Monte Carlo simulations and calculations of all the relevant background sources. The sources of background can be divided into external and internal ones. External background is generated by events originating from outside the liquid shielding, such as photons and neutrons from the Gran Sasso rock, muon interactions 
and muon induced activities. Internal background arises from residual impurities in the liquid nitrogen, in the steel vessel, in the crystal holder system, in the Ge crystals themselves and from activation of both liquid nitrogen and Ge crystals at the Earths surface.

For the simulation of muon showers, the external photon flux and the radioactive decay chains we used the GEANT3.21 package [12 extended for nuclear decays [13]. This version had already successfully been tested in establishing a quantitative background model for the Heidelberg-Moscow experiment [10].

In a first crude estimation the influence of the components expected to be dominant were studied [3,6]. In the simulation the setup consisted of a tank, 9 $\mathrm{m}$ in height and $9 \mathrm{~m}$ in diameter, with 288 naked enriched germanium crystals of $3.6 \mathrm{~kg}$ each, positioned in its center (this simulation has been performed in view of GENIUS as a neutrinoless double beta decay experiment with 1 ton of enriched ${ }^{76} \mathrm{Ge}$ ). From the background sources mentioned above, only the activities in the liquid nitrogen, steel vessel and muon showers were simulated.

For a more detailed study of the sensitivity of GENIUS in a first experimental phase, further simulations with a new, more accurate geometry have been carried out [14. The vessel is surrounded by a $2 \mathrm{~m}$ thick polyethylenefoam isolation, which is held by two $2 \mathrm{~mm}$ thick steel layers (constructional data from Messer-Griesheim). The nitrogen shielding is given by a cylindrical geometry of $12 \mathrm{~m}$ diameter and $12 \mathrm{~m}$ height with the crystals in its center. The simulated setup consisted of 5 natural Ge detectors integrated into a holder system of high molecular polyethylene. In comparison to the first simulation, the active mass reduces to approximately $14 \mathrm{~kg}$ and the anticoincidence of the detectors [3] will obviously be less effective.

\subsection{External background}

\subsubsection{Photon and neutron flux from the surroundings}

We simulated the influence of the photon flux with energies between $0-3$ $\mathrm{MeV}$ measured in hall $\mathrm{C}$ of the Gran Sasso laboratory [15]. This measurement is in good agreement with photon flux calculations by the Borexino Collaboration [16]. The main contributions are given in table 11. With a diameter of $12 \mathrm{~m}$ for the liquid shielding, we obtain a count rate of $4 \times 10^{-3}$ counts $/(\mathrm{kg} y$ $\mathrm{keV}$ ) in the energy region $0-100 \mathrm{keV}$.

The influence of the measured neutron flux in the Gran Sasso laboratory 117 was estimated. The $2 \mathrm{~m}$ polyethylene foam isolation $\left(\rho=0.03 \mathrm{~g} \mathrm{~cm}^{-3}\right)$ reduces the neutron flux for energies below $1 \mathrm{keV}$ by more than 5 orders of magnitude. Only about $3 \%$ of neutrons with energies between $1 \mathrm{keV}$ and 2.5 $\mathrm{MeV}$ will pass the polyethylene isolation, whereas for energies between 2.5 and $15 \mathrm{MeV}$ the overall flux is reduced by about $40 \%$. The neutron flux reaching the tank can be reduced by another two orders of magnitude by doping the polyethylene foam isolation with about $1.4 \mathrm{t}$ of boron. The flux of the ${ }^{7} \mathrm{Li}$ deexcitation gamma rays from the reaction $\mathrm{n}+{ }^{10} \mathrm{~B} \rightarrow \alpha+{ }^{7} \mathrm{Li}^{*}$, with an 
energy of $0.48 \mathrm{MeV}$, would be too low to reach the inner part of the liquid shielding. After the first meter of liquid nitrogen the total flux is reduced by another 4-5 orders of magnitude, therefore we simulated the neutron capture reactions randomly distributed in the first meter of the nitrogen shielding.

With the assumption that all neutrons reaching the nitrogen are thermalized and captured by the reactions ${ }^{14} \mathrm{~N}(\mathrm{n}, \mathrm{p}){ }^{14} \mathrm{C}^{*}$ and ${ }^{14} \mathrm{~N}(\mathrm{n}, \gamma){ }^{15} \mathrm{~N}^{*}$, a total of $4.4 \times 10^{7}$ neutron capture reactions per year have to be taken into account. The relevant contribution to the background comes from the deexcitation of the ${ }^{14} \mathrm{C}^{*}$ and ${ }^{15} \mathrm{~N}^{*}$ nuclei. The contribution of the $\beta$-decay of ${ }^{14} \mathrm{C}$ nuclei in the liquid nitrogen is negligible, since only low energy electrons $\left(\mathrm{E}_{\beta \max }=156\right.$ $\mathrm{keV}$ ) are emitted and the decay probability is very low due to the long half life $\left(10^{-4}\right.$ per year $)$.

Using the assumptions from above, the mean count rate in the low-energy region due to neutron capture reactions would be about $4 \times 10^{-4}$ counts $/(\mathrm{kg}$ y $\mathrm{keV})$.

\subsubsection{Activities induced by muons}

The muon flux in the Gran Sasso laboratory was measured to be $\phi_{\mu}=2.3 \times 10^{-4}$ $\mathrm{m}^{-2} \mathrm{~s}^{-1}$ with a mean energy of $\bar{E}_{\mu}=200 \mathrm{GeV}$ [15].

We simulated the effect of muon induced showers in the liquid nitrogen. With the aid of a muon veto in form of scintillators or gas counters on top of the tank, the total induced background can be drastically reduced. Here we assumed a veto efficiency of $96 \%$ as measured in a more shallow laboratory [18]. The count rate reached in the low-energy region is about $2 \times 10^{-3}$ counts $/ \mathrm{kg}$ $\mathrm{y} \mathrm{keV)}$. It can be further improved using the anticoincidence power of the 40 Ge detectors of the future dark matter setup among each other.

Besides muon showers, we have to consider muon induced nuclear disintegration and interactions due to secondary neutrons generated in the above reactions.

\section{Neutrons generated by cosmic muons}

The muon induced production of neutrons can be approximated by $\mathrm{A}_{n} \sim$ $3.2 \times 10^{-4}\left(\mathrm{~g}^{-1} \mathrm{~cm}^{2}\right)$, due to the $<E>^{0.75}$ dependence of the number of neutrons on the mean muon energy [19]. With the geometry of the tank $\mathrm{h}=$ $12 \mathrm{~m}, \mathrm{r}=6 \mathrm{~m}$, the density of nitrogen $\rho=0.808 \mathrm{~g} / \mathrm{cm}^{3}$ and the cited flux, a mean production rate of $\phi_{n \mu}=2.5 \times 10^{5}$ neutrons/year in the whole vessel is obtained. Table 2 gives the neutron induced reactions in the liquid nitrogen for neutron energies $<20 \mathrm{MeV}$ (based on all reactions found in [20]).

All of the produced nuclides are stable or short lived with the exception of ${ }^{14} \mathrm{C}$ and ${ }^{13} \mathrm{~N}$. The contribution of gammas from the excited ${ }^{14} \mathrm{C}^{*}$ nucleus corresponds to $10^{-3}$ counts $/(\mathrm{kg}$ y $\mathrm{keV})$ between $0-100 \mathrm{keV}$. The contribution from the $\beta^{-}$- particles with $\mathrm{E}_{\max }=0.16 \mathrm{MeV}$ is negligible due to the low decay probability of ${ }^{14} \mathrm{C}$. The production rate of ${ }^{13} \mathrm{~N}$ is $1 \times 10^{6}$ atoms per year in the whole tank. From $10^{6}$ simulated positrons with $\mathrm{E}_{\max }=1.2 \mathrm{MeV}\left(\beta^{+}\right.$-decay), 
corresponding to an exposure of about 1 year, only one event could be observed in the detectors. Therefore, the contribution of ${ }^{13} \mathrm{~N}$ to the background will be negligible.

\section{Negative muon capture}

A negative muon stopped in the liquid shielding can be captured by a nitrogen nucleus, leading to one of the reactions that are listed in table 3 . Estimations of the number of stopping muons in the nitrogen tank [21,22, 23] lead to 86 stopped muons per day. The rates of decaying and captured muons are shown in table 4 .

The derived production rates [24] for the various isotopes are listed in table 3. Only the isotopes ${ }^{14} \mathrm{C},{ }^{10} \mathrm{Be},{ }^{11} \mathrm{C}$ and ${ }^{10} \mathrm{C}$ can not be discriminated by muon anticoincidence, since their individual lifetime is too long. ${ }^{14} \mathrm{C}$ and ${ }^{10} \mathrm{Be}$ will not be seen in our detector due to their very low decay probabilities $\left(10^{-4}\right.$ and $10^{-10}$ per year) and low production rates. The contribution of ${ }^{10} \mathrm{C}$ and ${ }^{11} \mathrm{C}$, with a production rate of 117 atoms/year and 292 atoms/year, respectively, in the whole nitrogen tank, will be negligible. The gamma rays from the excited nuclei produced in the reactions can be discriminated by the anticoincidence shielding.

\section{Inelastic muon scattering}

Another way of producing radioactive isotopes in the liquid shielding are electromagnetic nuclear reactions of muons through inelastic scattering off nitrogen nuclei: $\mu+\mathrm{N} \rightarrow \mu^{\prime}+\mathrm{X}^{*}$. The only resulting isotopes with half lifes $>1 \mathrm{~s}$ are ${ }^{14} \mathrm{~N}(\gamma, \mathrm{n}){ }^{13} \mathrm{~N}$, with $\mathrm{T}_{1 / 2}=9.96 \mathrm{~m}$ and ${ }^{14} \mathrm{~N}(\gamma, \mathrm{tn}){ }^{10} \mathrm{C}$, with $\mathrm{T}_{1 / 2}=19.3 \mathrm{~s}$. The production rate per day for one isotope can be written as [25]: $\mathrm{R}\left(\mathrm{d}^{-1}\right)=$ $6 \times 10^{-2} \phi_{\mu}\left(\mathrm{d}^{-1} \mathrm{~m}^{-2}\right) \mathrm{N}_{T}(\mathrm{kt}) \sigma_{\mu}(\mu \mathrm{b}) / \mathrm{A}$, where $\phi_{\mu}$ is the flux of muons on the detector, $\mathrm{N}_{T}$ is the number of target nuclei, $\sigma_{\mu}$ the reaction cross section and A the atomic weight of the target nucleus. For our detector this yields $R\left(y^{-1}\right)=$ $45 \times \sigma_{\mu}(\mu \mathrm{b})$. With typical reaction cross sections of a few hundred $\mu \mathrm{b}$ [25.26], we obtain a production rate of $(5-10) \times 10^{3}$ atoms per year. A simulation of an activation time of ten years for both isotopes yields negligible count rates in comparison to contributions from other background components.

\subsection{Internal background}

\subsubsection{Intrinsic impurities in the nitrogen shielding, Ge crystals, holder sys- tem and steel vessel}

The assumed impurity levels for the liquid nitrogen are listed in table 5. For the ${ }^{238} \mathrm{U}$ and ${ }^{232} \mathrm{Th}$ decay chains they have already been reached by the Borexino experiment 27 for their liquid scintillator. Due to the very high cleaning efficiency of fractional distillation, it is conservative to assume that these requirements will also be fulfilled for liquid nitrogen. The ${ }^{238} \mathrm{U}$ and

${ }^{232}$ Th decay chains were simulated under the assumption that the chains are in 
secular equilibrium. The count rate due to ${ }^{238} \mathrm{U},{ }^{232} \mathrm{Th}$ and ${ }^{40} \mathrm{~K}$ contaminations of the liquid nitrogen is about $3.1 \times 10^{-3}$ in the energy region below $100 \mathrm{keV}$.

Measurements indicate that the ${ }^{222} \mathrm{Rn}$ contamination of freshly produced liquid nitrogen is in the range of $10 \mathrm{mBq} / \mathrm{m}^{3}$. After about a month it is reduced to $100 \mu \mathrm{Bq} / \mathrm{m}^{3}$ ( $\mathrm{T}_{1 / 2}=3.8$ days). Such a level could be maintained if the evaporated nitrogen is always replaced by Rn-pure nitrogen, previously stored in the underground. Surface emanations are reduced to a negligible level for cooled surfaces in direct contact with the liquid nitrogen. Non cooled surfaces have to be highly contamination free from ${ }^{222} \mathrm{Rn}$.

The mean count rate from the contamination of ${ }^{222} \mathrm{Rn}$ in the interesting region below $100 \mathrm{keV}$ is $10^{-3}$ counts/( $\mathrm{kg}$ y $\left.\mathrm{keV}\right)$ assuming an activity of 100 $\mu \mathrm{Bq} / \mathrm{m}^{3}$ in the liquid nitrogen.

For the intrinsic impurity concentration in Ge crystals we can give only upper limits from measurements with the detectors of the Heidelberg-Moscow experiment. We see a clear $\alpha$-peak in two of the enriched detectors at 5.305 $\mathrm{MeV}$, and an indication for the same peak in two other detectors. It originates from the decay of ${ }^{210} \mathrm{Po}$ (which decays with $99 \%$ through an $\alpha$-decay to ${ }^{206} \mathrm{~Pb}$ ) and is a sign for a ${ }^{210} \mathrm{~Pb}$ contamination of the detectors. However, it is very unlikely that the contamination is located inside the Ge-crystals, most probably it is located on the crystals surface at the inner contact.

Using three Ge detectors, we derive an upper limit at 90\% CL (after $19 \mathrm{~kg}$ y counting statistics) of $1.8 \times 10^{-15} \mathrm{~g} / \mathrm{g}$ for ${ }^{238} \mathrm{U}$ and $5.7 \times 10^{-15} \mathrm{~g} / \mathrm{g}$ for ${ }^{232} \mathrm{Th}$. Assuming these impurity concentrations, our simulations yield a count rate of about $10^{-2}$ counts/( $\mathrm{kg}$ y keV) for both ${ }^{238} \mathrm{U}$ and ${ }^{232} \mathrm{Th}$ decay chains. It is however secure to assume that these upper limits are very conservative and that the true contamination of HPGe is much lower. Special attention will have to be paid in order to avoid surface contaminations of the crystals.

An important open factor in the background spectrum is the effect of the holder-system. For the simulation we assumed the possibility to obtain a polyethylene with an impurity concentration of $10^{-13} \mathrm{~g} / \mathrm{g}$ for the $\mathrm{U} / \mathrm{Th}$ decay chains (this is a factor of 100 higher than the values reached for the organic liquid-scintillator by the Borexino collaboration [27]). Encouraging are the results already achieved by the SNO experiment [28], which developed an acrylic with current limits on ${ }^{232} \mathrm{Th}$ and ${ }^{238} \mathrm{U}$ contamination of $10^{-12} \mathrm{~g} / \mathrm{g}$. Since it is not yet sure that such a low contamination level will be reached for polyethylene, we are currently testing also other materials.

Assuming the above impurity level with the simulated geometry a count rate of $\sim 8 \times 10^{-4}$ counts/(kg y keV) in the energy region below $100 \mathrm{keV}$ from this component is reached. This result will be further improved by development of a new holder design using a minimized amount of material.

For the steel vessel an impurity concentration of $1 \times 10^{-8} \mathrm{~g} / \mathrm{g}$ for $\mathrm{U} / \mathrm{Th}$ was assumed (Borexino measured $5 \times 10^{-9} \mathrm{~g} / \mathrm{g}$ [16]). The contribution in the energy region $0-100 \mathrm{keV}$ is $1.5 \times 10^{-5}$ counts/( $\mathrm{kg}$ y $\left.\mathrm{keV}\right)$. Assuming an equal contamination for the polyethylene foam isolation as for steel, the contribution of both to the background is negligible. 


\subsubsection{Cosmic activation of the germanium crystals}

We have estimated the cosmogenic production rates of radioisotopes in the germanium crystals with the $\Sigma$ programme [29]. The programme was developed to calculate cosmogenic activations of natural germanium, enriched germanium and copper. It was demonstrated that it can reproduce the measured cosmogenic activity in the Heidelberg-Moscow experiment [10] within about a factor of two [30].

Assuming a production and transportation time of 10 days at sea level for the natural Ge detectors, and a deactivation time of three years, we obtain the radioisotope concentrations listed in table 6. All other produced radionuclides have much smaller activities due to their shorter half lifes.

The count rate below $11 \mathrm{keV}$ is dominated by $\mathrm{X}$-rays from the decays of ${ }^{68} \mathrm{Ge},{ }^{49} \mathrm{~V},{ }^{55} \mathrm{Fe}$ and ${ }^{65} \mathrm{Zn}$ (see table 6). Due to their strong contribution, the energy threshold of GENIUS would be at $11 \mathrm{keV}$, which is still acceptable (as can be seen from figure (1).

Between $11 \mathrm{keV}$ and $70 \mathrm{keV}$ the contribution from ${ }^{63} \mathrm{Ni}$ dominates due to the low $\mathrm{Q}-$ value $(66.95 \mathrm{keV})$ of the $\beta^{-}$-decay. Figure 目 shows the sum and the single contributions from the different isotopes. ${ }^{68} \mathrm{Ge}$ plays a special role. Since it can not be extracted by zone melting like all other, non-germanium isotopes, the starting activity would be in equilibrium with the production rate. With a half-life of $288 \mathrm{~d}$ it would by far dominate the other background components. A solution could be to process the germanium ore directly in the underground or to use high purity germanium which has already been stored for several years in an underground laboratory.

The sum of all contributions from the cosmogenic activation of the Ge crystals is $1.9 \times 10^{-2}$ counts $/(\mathrm{kg} \mathrm{y} \mathrm{keV})$ between $11-100 \mathrm{keV}$, for an activation time of 10 days at the Earths surface and a deactivation time of three years. Since this will be the dominant background component in the low energy region, special attention to short crystal exposure times at sea level is essential. The best solution would be to produce the detectors underground and to apply strong shielding during the transportation.

The two-neutrino accompanied double beta decay of ${ }^{76} \mathrm{Ge}$ is not negligible in spite of the low abundance $(7.8 \%)$ of this isotope in natural germanium. The contribution to the background after three years of measurement is shown in figure 6. Due to the already high statistics reached in the Heidelberg-Moscow experiment [10], the half life and spectral form of the decay are well known and a subtraction of this part raises no difficulties. The statistical error of the subtraction is not shown in figure $⿴$.

The cosmogenic activation of the Ge crystals in the Gran Sasso laboratory is negligible in comparison to the assumed activation scenario at sea level.

\subsubsection{Cosmic activation of the nitrogen at sea level}

An estimation of the production rates of long-lived isotopes in the nitrogen at sea level reveals the importance of ${ }^{7} \mathrm{Be},{ }^{10} \mathrm{Be},{ }^{14} \mathrm{C}$ and ${ }^{3} \mathrm{H}$. The neutron flux 
at sea level is $8.2 \times 10^{-3} \mathrm{~cm}^{-2} \mathrm{~s}^{-1}$ for neutron energies between $80 \mathrm{MeV}$ and 300 $\mathrm{MeV}$ [31]. Since we didn't find measurements of the cross sections of neutron induced spallation reactions in nitrogen, we assumed that at high neutron energies $\left(10^{2}-10^{4} \mathrm{MeV}\right)$ the cross sections are similar to the proton induced ones. For the reaction ${ }^{14} \mathrm{~N}(\mathrm{n}, \mathrm{t} \alpha \mathrm{n})^{7} \mathrm{Be}$ the cross section was measured to be $(9.0 \pm 2.1) \mathrm{mb}$ at $\mathrm{E}_{p}=450 \mathrm{MeV},(9.3 \pm 2.1) \mathrm{mb}$ at $\mathrm{E}_{p}=3000 \mathrm{MeV}$ by 32 and $(13.3 \pm 1.3) \mathrm{mb}$ at $\mathrm{E}_{p}=1600 \mathrm{MeV}$ by [33]. For the reaction ${ }^{14} \mathrm{~N}(\mathrm{n}, \alpha \mathrm{p}){ }^{10} \mathrm{Be}$ the measured cross sections are $(1.5 \pm 0.4) \mathrm{mb}$ at $\mathrm{E}_{p}=450 \mathrm{MeV},(2.6 \pm 0.6) \mathrm{mb}$ at $\mathrm{E}_{p}=3000 \mathrm{MeV}$ [32] and $(1.75 \pm 0.11) \mathrm{mb}$ at $\mathrm{E}_{p}=1600 \mathrm{MeV}$ [33].

Taking $10 \mathrm{mb}$ for the ${ }^{7}$ Be channel we obtain a production rate of $3.3 \times 10^{9} \mathrm{~d}^{-1}$ in the whole tank. This corresponds with a realistic 10 days sea level exposure after production by fractional distillation to $4 \times 10^{8}$ decays per day. The simulation of this activity yields a count rate of about 10 events/(kg y keV) in the energy region between $0-100 \mathrm{keV}$. This is three orders of magnitude higher than the required level. However, it can be expected, that a large fraction of ${ }^{7} \mathrm{Be}$ is removed from the liquid nitrogen at the cleaning process for $\mathrm{Rn}$ and that in addition by underground storage $\left(\mathrm{T}_{1 / 2}=53.3 \mathrm{~d}\right)$ the contribution of ${ }^{7} \mathrm{Be}$ is reduced to less than $10^{-2}$ events $/(\mathrm{kg}$ y $\mathrm{keV})$.

For ${ }^{10} \mathrm{Be}$, with $\sigma=2 \mathrm{mb}$, the production rate is $6.6 \times 10^{8} \mathrm{~d}^{-1}$, which is negligible due to the long half life of $\mathrm{T}_{1 / 2}=1.6 \times 10^{6} \mathrm{y}$.

Tritium in nitrogen can be produced in the following reactions: ${ }^{14} \mathrm{~N}(\mathrm{n}, \mathrm{t}){ }^{12} \mathrm{C}$, ${ }^{14} \mathrm{~N}(\mathrm{n}, \mathrm{t} 2 \alpha){ }^{4} \mathrm{He},{ }^{14} \mathrm{~N}(\mathrm{n}, \mathrm{t} \alpha \mathrm{n}){ }^{7} \mathrm{Be}$ and ${ }^{14} \mathrm{~N}(\mathrm{n}, \mathrm{tn}){ }^{11} \mathrm{C}$. The cross section for the production by ${ }^{14} \mathrm{~N}(\mathrm{n}, \mathrm{t}){ }^{12} \mathrm{C}$ was measured to be $40 \mathrm{mb}$ [34]. For a rough estimation, we assumed the same cross section for the other reaction as for the production of ${ }^{7} \mathrm{Be}$ to be $10 \mathrm{mb}$. The total production rate of tritium corresponds to $2.3 \times 10^{10} \mathrm{~d}^{-1}$. With $\mathrm{T}_{1 / 2}=12.33 \mathrm{y}$, the activity after 10 days exposure at sea level would be $3.5 \times 10^{7} \mathrm{~d}^{-1}$. We simulated $10^{10}$ decays randomly distributed in the nitrogen tank. No events were detected mainly due to the absorption in the dead layer of the $\mathrm{p}$-type Ge detectors.

The muon flux at sea level is $1.6 \times 10^{7} \mathrm{~m}^{-2} \mathrm{~d}^{-1}$. The only long-lived isotopes which are produced by inelastic muon scattering are ${ }^{13} \mathrm{~N}$ and ${ }^{10} \mathrm{C}$, with a production rate of about $3.7 \times 10^{7}$ atoms/days (taking $\sigma=500 \mu \mathrm{b}$ for both reactions). However, ${ }^{13} \mathrm{~N}$ and ${ }^{10} \mathrm{C}$ are of no relevance due to the short half lifes of $9.96 \mathrm{~m}$ and $19.3 \mathrm{~s}$, respectively.

The isotopes produced through negative muon capture with half lifes $>$ 1s are ${ }^{14} \mathrm{C},{ }^{10} \mathrm{Be},{ }^{11} \mathrm{C}$ and ${ }^{10} \mathrm{C}$ (see also table 3). Again, the number of ${ }^{11} \mathrm{C}$ and ${ }^{10} \mathrm{C}$ isotopes are soon reduced to a negligible level due to their short half lifes. The production rate for the whole tank for ${ }^{14} \mathrm{C}$ is $8 \times 10^{6} \mathrm{~d}^{-1}$ and $4 \times 10^{5} \mathrm{~d}^{-1}$ for ${ }^{10} \mathrm{Be}$, which have to be added to the production rates by neutron capture or spallation reactions.

For the production of ${ }^{14} \mathrm{C}$ due to the ${ }^{14} \mathrm{~N}(\mathrm{n}, \mathrm{p}){ }^{14} \mathrm{C}$ capture reaction, three neutron sources at sea level are relevant. The flux of secondary cosmic ray neutrons with energies between a few $\mathrm{keV}$ and $20 \mathrm{MeV}$ is about $2 \times 10^{-2} \mathrm{~cm}^{-2} \mathrm{~s}^{-1} 31$. These neutrons penetrate the wall of the transportation tank and are captured in the liquid nitrogen. For a tank surface of $678 \mathrm{~m}^{2}$, about $1.3 \times 10^{5} \mathrm{~s}^{-1}$ neutrons 
are expected. The second component are neutrons produced in fast neutron spallation reactions in the liquid nitrogen. The production rate of these neutrons is $2 \times 10^{4} \mathrm{~s}^{-1}$ in the nitrogen tank [35. The third component are neutrons produced in muon reactions, which correspond to $0.85 \times 10^{3} \mathrm{~s}^{-1}$ [35]. Thus the total flux at sea level is about $1.5 \times 10^{4} \mathrm{~s}^{-1}$. Assuming that every neutron is captured in the nitrogen, yielding a ${ }^{14} \mathrm{C}$ nucleus, the production rate of ${ }^{14} \mathrm{C}$ is about $1.3 \times 10^{10} \mathrm{~d}^{-1}$. For a production and transportation time of ten days, the simulation yields less than $10^{-4}$ counts/( $\mathrm{kg} \mathrm{keV} \mathrm{y)} \mathrm{in} \mathrm{the} \mathrm{relevant} \mathrm{energy} \mathrm{re-}$ gion. Through the purification of the nitrogen this contribution will be further reduced.

\subsection{Sum spectrum from simulations}

In table 7 the components discussed so far are listed and summed up. Not included in the table are the contributions from the intrinsic impurities in the Ge crystals and from the ${ }^{7}$ Be activation of the liquid nitrogen during its transportation at sea level. For the Ge-crystals we have only very conservative upper values for their true contamination, which is expected to be much lower (see Section 3.2.1). Regarding the ${ }^{7}$ Be contamination of liquid nitrogen, we are confident that the cleaning efficiency is high enough in order to reduce this contribution to a negligible level. Assuming a background as stated above, we will achieve a mean count rate in the interesting region of about $3.1 \times 10^{-2}$ events/(kg y keV). This means a further reduction of background in comparison to our best measurement (about 20 counts/(kg y keV) below $100 \mathrm{keV}$ [9]) by about 3 orders of magnitude.

In figure 6 the spectra of individual contributions and the summed up total background spectrum are shown. As mentioned before, the low energy spectrum is dominated by events originating from the cosmogenic activation of the Ge crystals at the Earths surface. Production of the detectors underground would significantly reduce this contribution.

\section{Conclusion and Outlook}

After a first study of the dominant background sources in the GENIUS experiment [3.6], we performed a more detailed investigation of the background in the energy region relevant for the direct detection of WIMPs. At the same time a technical study with three naked Ge crystals in a common crystal holder immersed in liquid nitrogen was carried out.

We simulated an exact tank design with five natural germanium detectors supported by a holder system of high molecular polyethylene. The measured photon flux in the Gran Sasso laboratory with photon energies between 0 and $3 \mathrm{MeV}$ was simulated for all the dominant radioisotopes. The influence of neutrons from the natural radioactivity of the rocks was estimated under the conservative assumption that every neutron reaching the liquid shielding 
is captured leading to excited ${ }^{14} \mathrm{C}^{*}$ and ${ }^{15} \mathrm{~N}^{*}$ nuclei. We simulated the radon contamination of the nitrogen and studied the activation of the shielding by muon induced nuclear disintegration and by secondary neutrons from muon interactions. At the same time we calculated the activation of the Ge crystals and of the liquid nitrogen during the period of their exposure at sea level.

The obtained count rates and activation levels confirm the possibility to achieve a background count rate of $10^{-2}$ counts $/ \mathrm{kg} \mathrm{keV}$ y in the energy region below $100 \mathrm{keV}$.

For this, we need only fairly standard arrangements for the setup, like a boron implanted polyethylene foam isolation or a neutron absorption film, an anticoincidence shield for muons (scintillators on top of the setup) and a nitrogen-cleaning device (eventually with nitrogen recycling).

Besides that, we need more sensitive measurements of the contamination level of materials to hold the Ge-crystals and the Rn contamination of liquid nitrogen. Both measurements are underway. Furthermore, surface contamination of the crystals and the supporting structure needs high attention.

Reaching the background level aimed at, the GENIUS project could bring a large decisive progress in the field of direct dark matter search. It could probe a major part of the SUSY-WIMP parameter space interesting for the detection of neutralinos, thus possibly deciding whether or not neutralinos are the major component of the dark matter in our Galaxy.

\section{Acknowledgments}

L.B. is supported by the Graduiertenkolleg of the University of Heidelberg. She would like to thank Y. Declais for helpful discussions.

\section{References}

[1] H.V. Klapdor-Kleingrothaus in Proceedings of the First International Conference on Particle Physics Beyond the Standard Model, Castle Ringberg, Germany, 8-14 June 1997, edited by H.V. Klapdor-Kleingrothaus and H. Päs, IOP Bristol, (1998) 485-531

[2] H.V. Klapdor-Kleingrothaus, M. Hirsch, Z. Phys. A 359, (1997) 351

[3] H.V. Klapdor-Kleingrothaus, J. Hellmig, M. Hirsch, J. Phys. G 24, (1998) 483

[4] H.V. Klapdor-Kleingrothaus, Y. Ramachers, Europ. J. Phys. A 3, (1998) 85-92

[5] G. Heusser, Ann. Rev. Nucl. Part. Sci. 45, (1995) 543

[6] J. Hellmig, H.V. Klapdor-Kleingrothaus, Z. Phys. A 359, (1997) 361

[7] G. Jungman, M. Kamionkowski, K. Griest, Phys. Rep. 267, (1996) 195

[8] V. Bednyakov, H.V. Klapdor-Kleingrothaus, S. Kovalenko, Y. Ramachers, Z. Phys. A 357 (1997) 339 
[9] L. Baudis et al. (Heidelberg-Moscow collaboration), accepted for publication in Phys. Rev. D

[10] Heidelberg-Moscow Collaboration, M. Günther et al., Phys. Rev. D 55, 54 (1997) and L. Baudis et al., Phys. Lett. B 407, (1997) 219

[11] G. F. Knoll, Radiation Detection and Measurement, 2. ed., Wiley, 1989

[12] GEANT3.21 Detector description and simulation tool, Geneva 1993

[13] A. Müller, PhD Thesis, University of Heidelberg 1993

[14] B. Majorovits, Diploma thesis, University of Heidelberg 1998, unpublished.

[15] C. Arpesella, Nucl. Phys. A 28 (1992) 420-424

[16] Borexino Collaboration, Proposal for a real time detector for low energy solar neutrinos, Dept. of Physics of the University of Milano (1991).

[17] P. Belli et al., Nuovo Cim., 101A, (1989) 959

[18] G. Heusser, Nucl. Instr. and Meth. in Phys. Res. B 58(1991) 79

[19] M. Aglietta et al., Il Nuovo Cimento C 12 (1989) 467

[20] V. McLane, C.L. Dunford, P.F. Rose, Neutron cross sections, Volume 2, Academic Press Inc., 1988

[21] L. Bergamasco et al., Il Nuovo Cimento A 67, (1982) 255

[22] Th. Gaisser, Cosmic Rays and Particle Physics, Cambridge University Press, 1990.

[23] W. Lohmann, R. Kopp, R. Voss, Energy loss of muons in the energy range 1-10000 GeV, CERN 85-03, Geneva 1985.

[24] S. Charalambus, Nucl. Phys. A 166 (1971) 145

[25] J.S. O’Connell, F.J. Schima, Phys. Rev. D 38 (1988) 2277

[26] V. di Napoli, Phys. Rev. C 8 (1973) 206

[27] G. Alimonti et al. (BOREXINO Collaboration), Astropart. Phys., 8 (1998) 141

[28] SNO Collaboration, Proceedings of the 4th International Solar Neutrino Conference (Heidelberg, April 8-11 1997), edited by W. Hampel, (1997) 210

[29] J. Bockholt, PhD Thesis, University of Heidelberg 1994

[30] B. Maier, PhD Thesis, University of Heidelberg 1996

[31] O.C. Allkofer, P.K. Grieder, Cosmic Rays on Earth, Fachinformations-zentrum Karlsruhe, 1983

[32] J.L. Reyss, Y. Yokoyama and F. Guichard, Earth and Plan. Sci. Let. 53 (1981) 203 
[33] R. Michel et al., NIM B 103 (1995) 183

[34] G.P. Kincaid, E. R. Ibert jun., Nature 226 (1970) 139

[35] D. Lal, Ann. Rev. Earth Planet. Sci. 16 (1988) 355-388

[36] R. Bernabei et al., Nucl. Phys. B 70 (Proc. Suppl) (1998) 79

[37] L. Baudis et al., Nucl. Instrum. Methods A 385, (1997) 265

[38] D. S. Akerib et al., CDMS Collaboration, Nucl. Phys. B 70 (Proc. Suppl) (1998) 64

[39] M. Bühler et al., NIM A 370 (1996) 237

[40] R. Bernabei et al., ROM2F/98/34, August (1998) 


\begin{tabular}{lcc}
\hline Isotope & Energy $[\mathrm{keV}]$ & Flux $\left[\mathrm{m}^{-2} \mathrm{~d}^{-1}\right]$ \\
\hline${ }^{40} \mathrm{~K}$ & 1460 & $3.8 \times 10^{7}$ \\
\hline${ }^{214} \mathrm{~Pb}$ & 295.2 & $0.8 \times 10^{7}$ \\
${ }^{214} \mathrm{~Pb}$ & 352 & $1.8 \times 10^{7}$ \\
${ }^{214} \mathrm{Bi}$ & 609.3 & $2.9 \times 10^{7}$ \\
${ }^{214} \mathrm{Bi}$ & 1120.3 & $1.4 \times 10^{7}$ \\
${ }^{214} \mathrm{Bi}$ & 1764.5 & $1.7 \times 10^{7}$ \\
\hline${ }^{208} \mathrm{Tl}$ & 2614.5 & $1.35 \times 10^{7}$ \\
\hline
\end{tabular}

Table 1

Simulated components of the gamma ray flux from natural radioactivity in the Gran Sasso Laboratory (from 15]).

\begin{tabular}{lcc}
\hline Reaction & $\mathrm{T}_{1 / 2}$ of the product & Decay energy \\
\hline${ }^{14} \mathrm{~N}(\mathrm{n}, \mathrm{p}){ }^{14} \mathrm{C}$ & $\mathrm{T}_{1 / 2}=5.7 \times 10^{3} \mathrm{y}$ & $\mathrm{E}_{\beta^{-}}=0.16 \mathrm{MeV}$ \\
${ }^{14} \mathrm{~N}(\mathrm{n}, \gamma){ }^{15} \mathrm{~N}$ & stable & \\
${ }^{14} \mathrm{~N}(\mathrm{n}, 2 \mathrm{n}){ }^{13} \mathrm{~N}$ & $\mathrm{~T}_{1 / 2}=9.96 \mathrm{~m}$ & $\mathrm{E}_{\beta^{+}}=1.2 \mathrm{MeV}$ \\
${ }^{14} \mathrm{~N}(\mathrm{n}, \alpha){ }^{11} \mathrm{~B}$ & stable & \\
${ }^{14} \mathrm{~N}(\mathrm{n}, \mathrm{t})^{12} \mathrm{C}$ & stable & \\
${ }^{14} \mathrm{~N}(\mathrm{n}, 2 \alpha){ }^{7} \mathrm{Li}$ & stable & \\
\hline
\end{tabular}

Table 2

Neutron interactions in the liquid nitrogen for neutron energies $<20 \mathrm{MeV}$. 


\begin{tabular}{lccc}
\hline Reaction & $\mathrm{T}_{1 / 2}$ & Decay energy & Rate $\left[\mathrm{y}^{-1}\right]$ \\
\hline${ }^{14} \mathrm{~N}\left(\mu, \nu_{\mu}\right){ }^{14} \mathrm{C}$ & $\mathrm{T}_{1 / 2}=5.7 \times 10^{4} \mathrm{y}$ & $\mathrm{E}_{\beta^{-}}=0.16 \mathrm{MeV}$ & 584 \\
${ }^{14} \mathrm{~N}\left(\mu, \nu_{\mu} \alpha\right)^{10} \mathrm{Be}$ & $\mathrm{T}_{1 / 2}=1.6 \times 10^{10} \mathrm{y}$ & $\mathrm{E}_{\beta^{-}}=0.6 \mathrm{MeV}$ & 29 \\
${ }^{14} \mathrm{~N}\left(\mu, \nu_{\mu} \mathrm{p}\right){ }^{13} \mathrm{~B}$ & $\mathrm{~T}_{1 / 2}=17.33 \mathrm{~ms}$ & $\mathrm{E}_{\beta^{-}}=13.4 \mathrm{MeV}$ & 116 \\
${ }^{14} \mathrm{~N}\left(\mu, \nu_{\mu} \mathrm{n}\right){ }^{13} \mathrm{C}$ & stable & & 3798 \\
${ }^{14} \mathrm{~N}\left(\mu, \nu_{\mu} \alpha \mathrm{n}\right){ }^{9} \mathrm{Be}$ & stable & & 17 \\
${ }^{14} \mathrm{~N}\left(\mu, \nu_{\mu} \alpha \mathrm{p}\right){ }^{9} \mathrm{Li}$ & $\mathrm{T}_{1 / 2}=178 \mathrm{~ms}$ & $\mathrm{E}_{\beta^{-}}=13.6 \mathrm{MeV}$ & 0.6 \\
${ }^{14} \mathrm{~N}\left(\mu, \nu_{\mu} 2 \mathrm{n}\right){ }^{12} \mathrm{C}$ & stable & & 1168 \\
${ }^{14} \mathrm{~N}\left(\mu, \nu_{\mu} 3 \mathrm{n}\right){ }^{11} \mathrm{C}$ & $\mathrm{T}_{1 / 2}=20.38 \mathrm{~m}$ & $\mathrm{E}_{\beta^{-}}=13.4 \mathrm{MeV}, \mathrm{E}_{\gamma}=4.44 \mathrm{MeV}$ & 292 \\
${ }^{14} \mathrm{~N}\left(\mu, \nu_{\mu} 4 \mathrm{n}\right){ }^{10} \mathrm{C}$ & $\mathrm{T}_{1 / 2}=19.3 \mathrm{~s}$ & $\mathrm{E}_{\beta^{+}}=1.9 \mathrm{MeV}$ & 117 \\
\hline Table 3 & & &
\end{tabular}

Spallation reactions from muon capture.

Table 4

\begin{tabular}{lcc}
\hline Muon flux & $124 \mathrm{~h}^{-1}$ & \\
\hline Stopped muons & $86 \mathrm{~d}^{-1}$ & \\
\hline Decaying muons & $\mu^{+}$ & $\mu^{-}$ \\
& $50 \mathrm{~d}^{-1}$ & $20 \mathrm{~d}^{-1}$ \\
\hline Captured muons & $\mu^{+}$ & $\mu^{-}$ \\
& 0 & $16 \mathrm{~d}^{-1}$ \\
\hline
\end{tabular}

Muon flux, stopped, captured and decaying muons in the nitrogen shielding of the Genius detector.

Table 5

\begin{tabular}{lcc}
\hline Source & Radionuclide & Purity \\
\hline Nitrogen & ${ }^{238} \mathrm{U}$ & $1 \times 10^{-15} \mathrm{~g} / \mathrm{g}$ \\
& ${ }^{232} \mathrm{Th}$ & $5 \times 10^{-15} \mathrm{~g} / \mathrm{g}$ \\
& ${ }^{40} \mathrm{~K}$ & $1 \times 10^{-15} \mathrm{~g} / \mathrm{g}$ \\
\hline Steel vessel & $\mathrm{U} / \mathrm{Th}$ & $1 \times 10^{-8} \mathrm{~g} / \mathrm{g}$ \\
\hline
\end{tabular}

Restrictions in radioactive purity of single components for the liquid nitrogen. 


\begin{tabular}{lccc}
\hline Isotope & Decay mode, $\mathrm{T}_{1 / 2}$ & Energy $[\mathrm{keV}]$ & Activity $\left[\mu{\left.\mathrm{Bq} \mathrm{kg}^{-1}\right]}^{{ }^{49} \mathrm{~V}}\right.$ \\
${ }^{54} \mathrm{EC}, 330 \mathrm{~d}$ & no $\gamma, \mathrm{E}\left(\mathrm{K}_{\alpha}{ }^{49} \mathrm{Ti}\right)=4.5$ & 0.17 \\
${ }^{55} \mathrm{Fe}$ & $\mathrm{EC}, 312.2 \mathrm{~d}$ & $\mathrm{E}_{\gamma}=1377.1 \mathrm{E}\left(\mathrm{K}_{\alpha}{ }^{54} \mathrm{Cr}\right)=5.99$ & 0.20 \\
${ }^{55}, 2.73 \mathrm{a}$ & no $\gamma, \mathrm{E}\left(\mathrm{K}_{\alpha}{ }^{55} \mathrm{Mn}\right)=5.9$ & 0.18 \\
${ }^{57} \mathrm{Co}$ & $\mathrm{EC}, 271.3 \mathrm{~d}$ & $136.5(99.82 \%) \mathrm{E}\left(\mathrm{K}_{\alpha}{ }^{57} \mathrm{Fe}\right)=7.1$ & 0.18 \\
${ }^{60} \mathrm{Co}$ & $\beta^{-}, 5.27 \mathrm{a}$ & $318(99.88 \%), \mathrm{E}_{\gamma 1,2}=1173.24,1332.5$ & $1.2 \times 10^{-2}$ \\
${ }^{63} \mathrm{Ni}$ & $\beta^{-}, 100.1 \mathrm{a}$ & $\mathrm{E}_{\beta^{-}}=66.95 \mathrm{no} \gamma$ & 1.14 \\
${ }^{65} \mathrm{Zn}$ & $\mathrm{EC}, 244.3 \mathrm{~d}$ & $\mathrm{E}_{\gamma}=1115.55(50.6 \%), \mathrm{E}\left(\mathrm{K}_{\alpha}{ }^{65} \mathrm{Cu}\right)=8.9$ & 2.56 \\
${ }^{68} \mathrm{Ge}$ & $\mathrm{EC}, 288 \mathrm{~d}$ & no $\gamma, \mathrm{E}\left(\mathrm{K}_{\alpha}{ }^{68} \mathrm{Ga}\right)=10.37, \mathrm{Q}_{E C}\left({ }^{68} \mathrm{Ga}\right)=2921$ & \\
\hline
\end{tabular}

Table 6

Cosmogenic produced isotopes in the Ge crystals for an exposure time at sea level of 10 days and for 3 years deactivation time. 


\begin{tabular}{|c|c|c|}
\hline Source & Component & Count rate $(11-100 \mathrm{keV})[$ counts $/(\mathrm{kg} \mathrm{y} \mathrm{keV})]$ \\
\hline Nitrogen & ${ }^{238} \mathrm{U}$ & $2 \times 10^{-3}$ \\
\hline \multirow[t]{3}{*}{ intrinsic } & ${ }^{232} \mathrm{Th}$ & $1 \times 10^{-3}$ \\
\hline & ${ }^{40} \mathrm{~K}$ & $1 \times 10^{-4}$ \\
\hline & ${ }^{222} \mathrm{Rn}$ & $1 \times 10^{-3}$ \\
\hline $\mathrm{N}$ activation & ${ }^{14} \mathrm{C}$ & $1 \times 10^{-4}$ \\
\hline Steel vessel & $\mathrm{U} / \mathrm{Th}$ & $1.5 \times 10^{-5}$ \\
\hline Holder system & $\mathrm{U} / \mathrm{Th}$ & $8 \times 10^{-4}$ \\
\hline \multirow[t]{5}{*}{ Surrounding } & Gammas & $4 \times 10^{-3}$ \\
\hline & Neutrons & $4 \times 10^{-4}$ \\
\hline & Muon shower & $2 \times 10^{-3}$ \\
\hline & $\mu \rightarrow \mathrm{n}$ & $1 \times 10^{-3}$ \\
\hline & $\mu \rightarrow$ capture & $<<1 \times 10^{-4}$ \\
\hline Cosmogenic & ${ }^{54} \mathrm{Mn}$ & $3 \times 10^{-3}$ \\
\hline activities & ${ }^{57} \mathrm{Co}$ & $1 \times 10^{-3}$ \\
\hline \multirow[t]{4}{*}{ in the crystals } & ${ }^{60} \mathrm{Co}$ & $4 \times 10^{-3}$ \\
\hline & ${ }^{63} \mathrm{Ni}$ & $8 \times 10^{-3}$ \\
\hline & ${ }^{65} \mathrm{Zn}$ & $2 \times 10^{-3}$ \\
\hline & ${ }^{68} \mathrm{Ge}$ & $1 \times 10^{-3}$ \\
\hline Total & & $3.1 \times 10^{-2}$ counts $/(\mathrm{kg}$ y $\mathrm{keV})$ \\
\hline
\end{tabular}

Table 7

Summation of background components in the region $0 \mathrm{keV}-100 \mathrm{keV}$. 


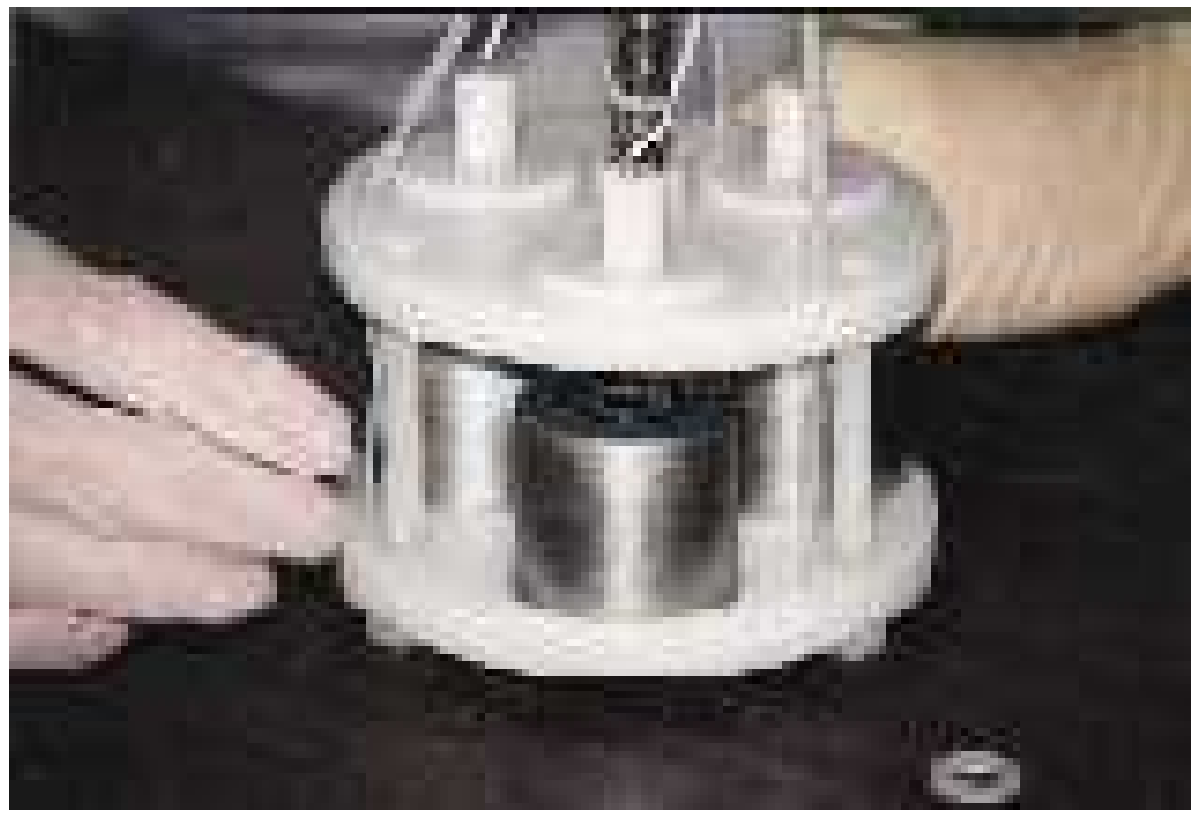

Fig. 1. The three-crystal holder-system with germanium crystals mounted shortly before cooling. Some crystal-to-FET cables can be seen. 


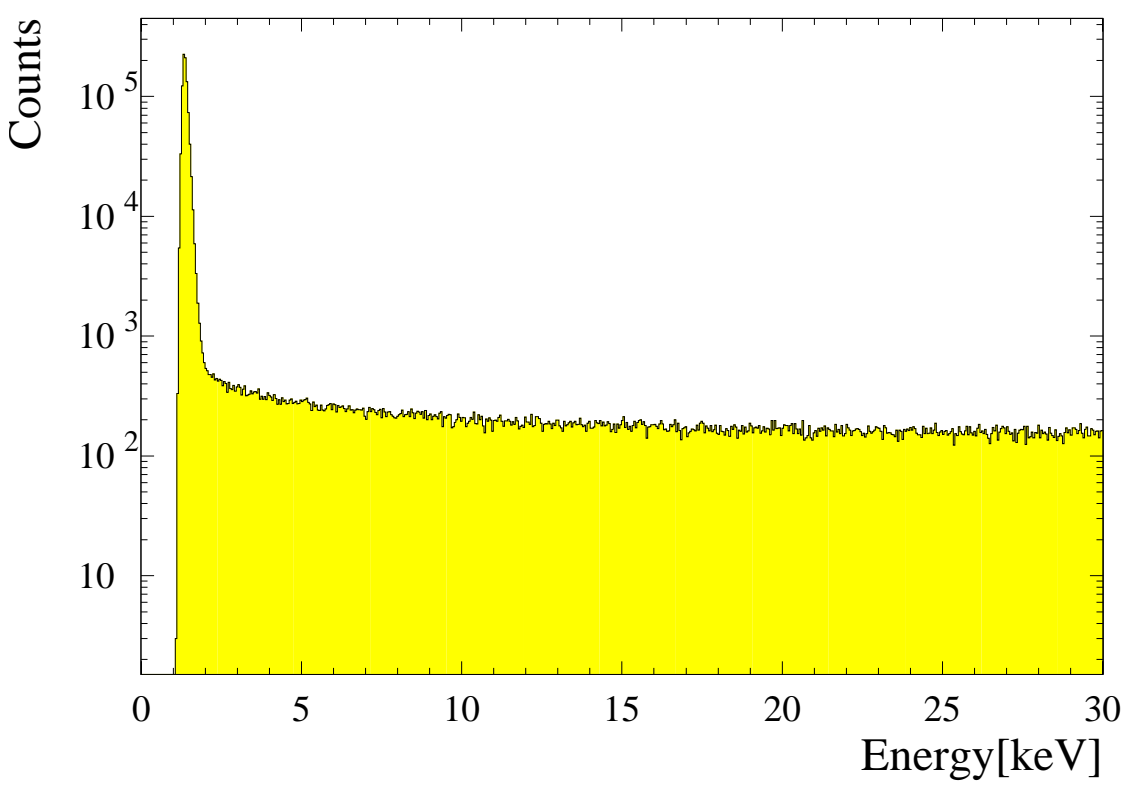

Fig. 2. Background spectrum of a naked, unshielded Ge crystal in liquid nitrogen. Note the low energy threshold of $2 \mathrm{keV}$ of the detector.

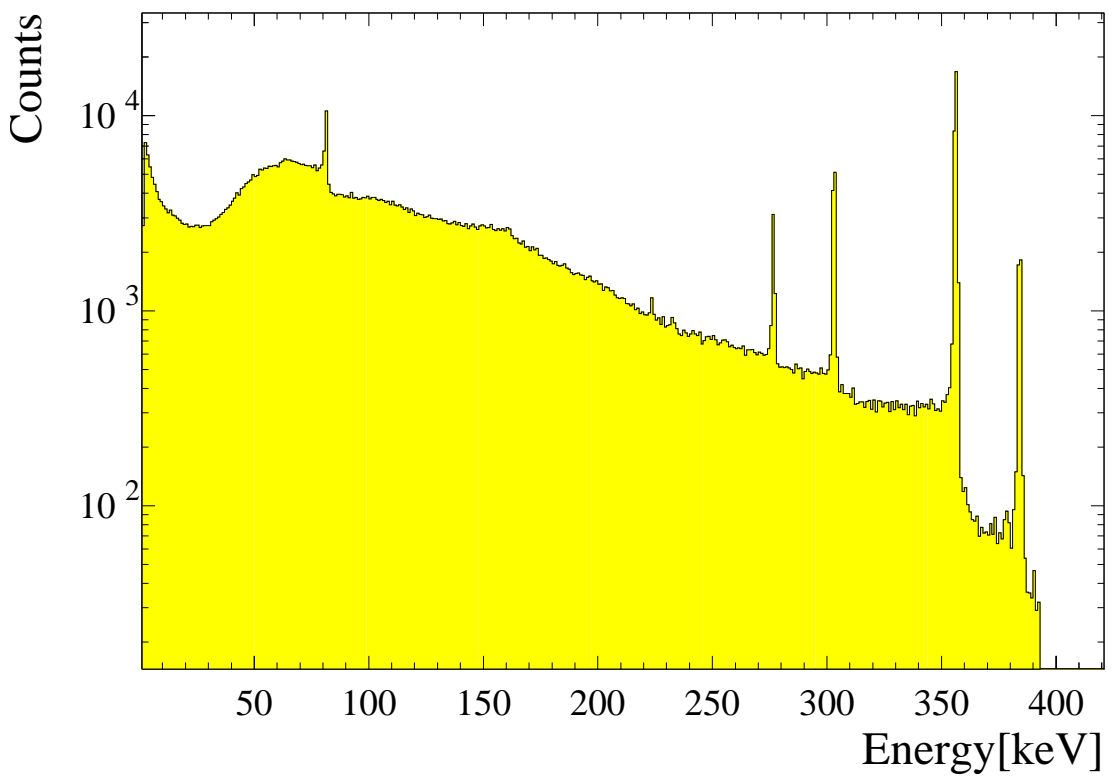

Fig. 3. Calibration ${ }^{133} \mathrm{Ba}$ spectrum of a naked Ge crystal in liquid nitrogen. The energy resolution is $1 \mathrm{keV}$ at $300 \mathrm{keV}$. 


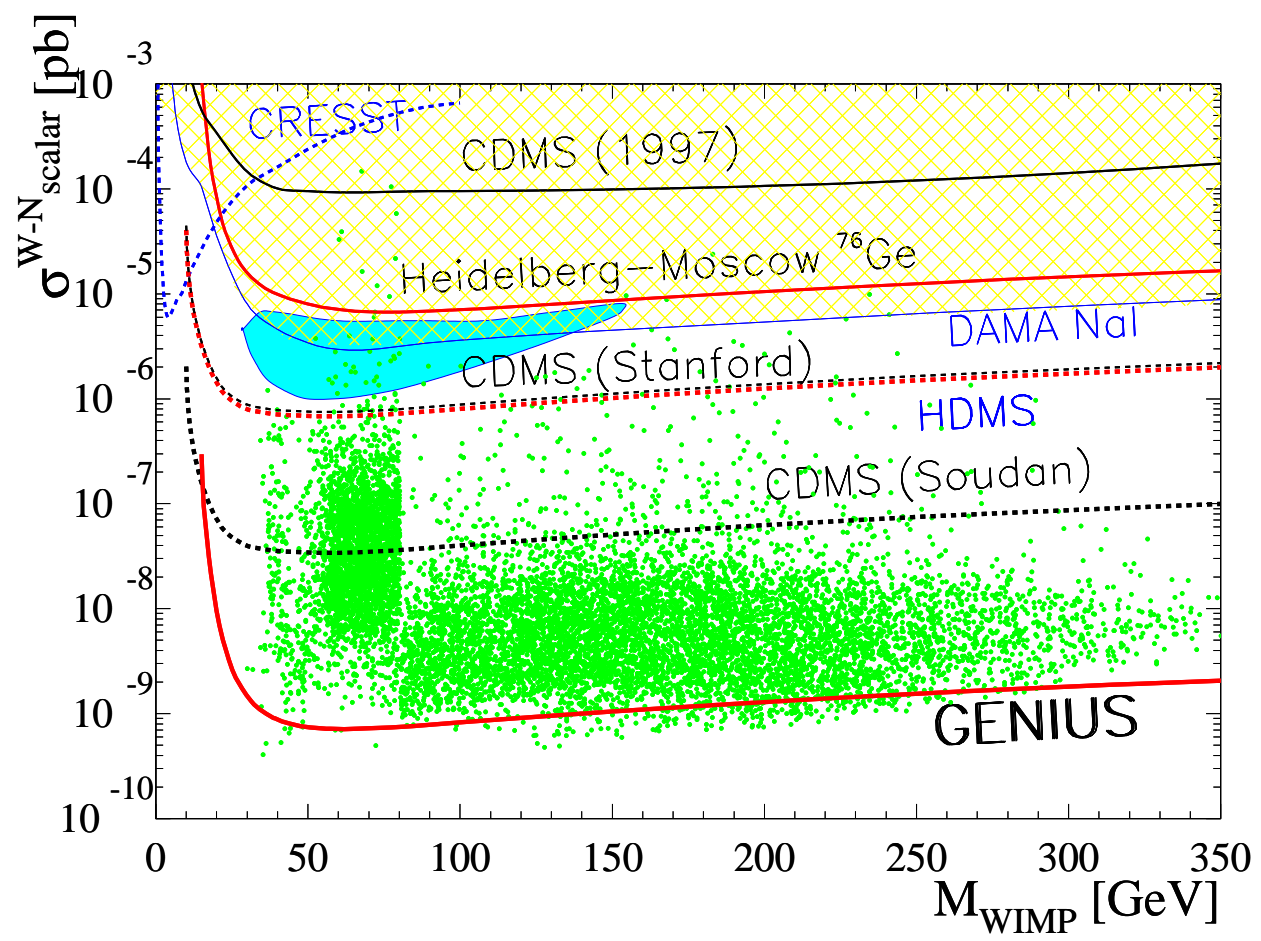

Fig. 4. WIMP-nucleon cross section limits as a function of the WIMP mass. The hashed region is excluded by the Heidelberg-Moscow [9] and the DAMA experiment [36], the dashed lines are expectations for recently started or future experiments, like HDMS [37], CDMS [38] and CRESST [39]. The filled contour represents the $2 \sigma$ evidence region of the DAMA experiment [40]. The solid line is the expectation for the GENIUS project with a background level of 0.01 counts/(keV $\mathrm{kg} y)$ and an energy threshold of $11 \mathrm{keV}$. The experimental limits are compared to expectations (scatter plot) for WIMP-neutralinos calculated in the MSSM framework with non-universal scalar mass unification [8]. 


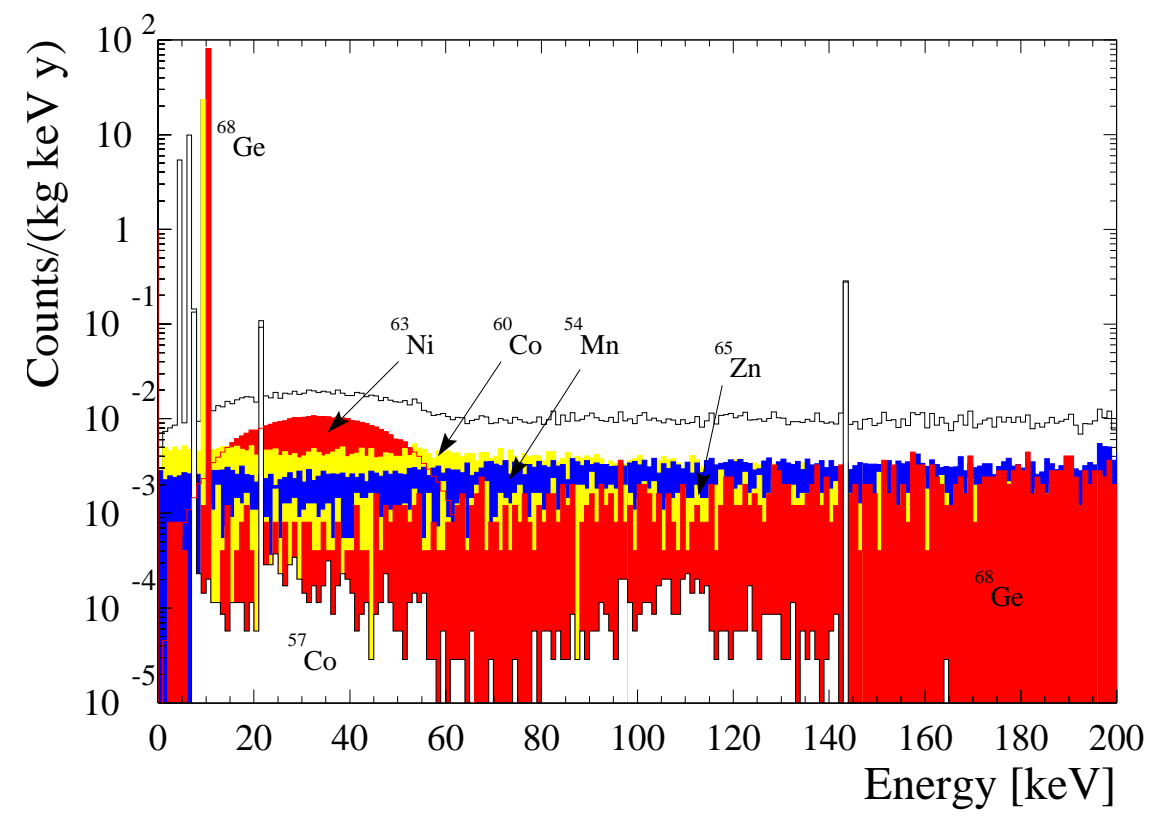

Fig. 5. Background originating from cosmic activation of the Ge crystals at sea level with 10 days exposure and 3 years deactivation.

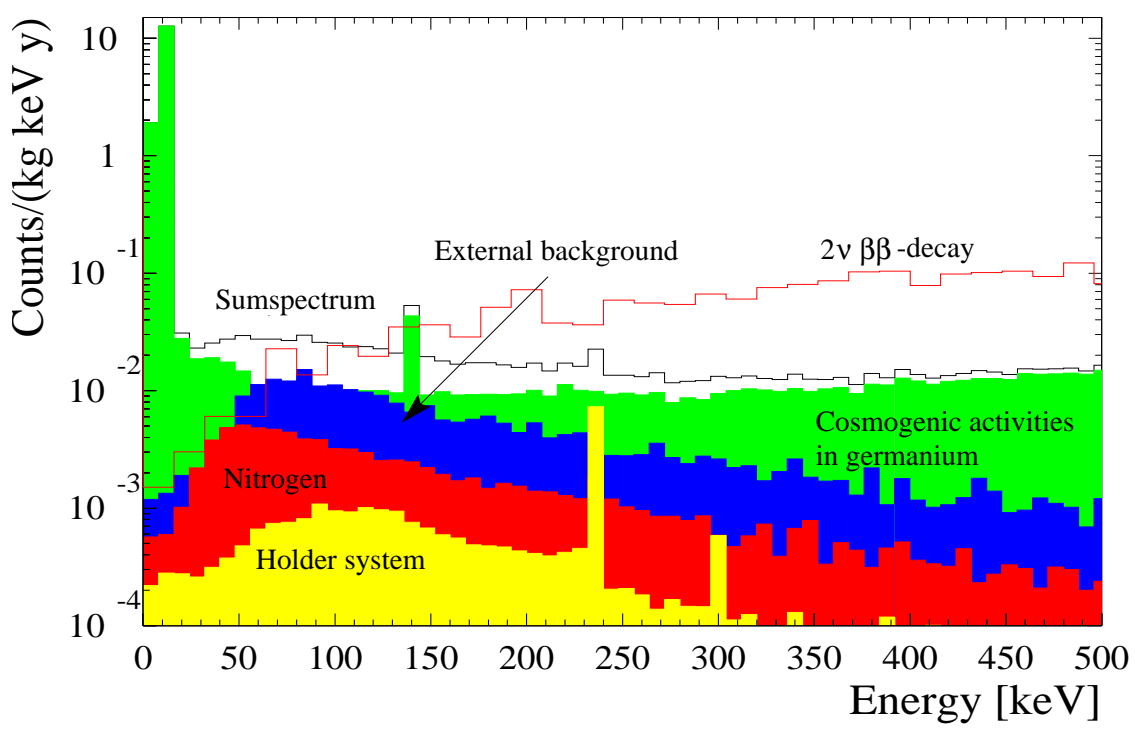

Fig. 6. Simulated spectra of the dominant background sources for a nitrogen tank of $12 \mathrm{~m}$ diameter. Shown are the contributions from the tank walls, the detector holder system, from neutron capture in the nitrogen, from natural radioactivity and from the ${ }^{222} \mathrm{Rn}$ contamination of the nitrogen. The solid line represents the sum spectrum of all the simulated components (note the different channel binning compared to figure 5). 